\title{
Quantification of Retinopathy of Prematurity via Vessel Segmentation
}

\author{
Julien Jomier ${ }^{1}$, David K. Wallace ${ }^{2}$, and Stephen R. Aylward ${ }^{1}$ \\ 1 Computer-Aided Diagnosis and Display Lab, Department of Radiology \\ \{jomier, aylward\}@unc. edu \\ 2 Department of Opthalmology \\ The University of North Carolina at Chapel Hill \\ 27599 Chapel Hill, USA \\ dkwallac@med.unc .edu
}

\begin{abstract}
Retinopathy of prematurity is a disease that affects the eyes of many babies who are prematurely born. If the retinopathy is not detected in the days following birth blindness may occur. Studies have demonstrated that by observing the blood vessels within the retina, the disease can be quantified in an early stage and early treatment can save the baby's eyes. We have developed a new tool to assess retinopathy of prematurity. Our technique captures images of the retina to extract and quantify both tortuosity and dilation of blood vessels. Our approach demonstrates a $80 \%$ sensitivity and $92 \%$ specificity in the prediction of retinopathy compared to experts and shows a significant reduced diagnosis time and clinical integration via speech recognition and glare detection.
\end{abstract}

\section{Introduction}

Retinopathy of prematurity (ROP) is an eye disease that occurs in some prematurely born babies. The last 12 weeks of a full-term delivery, from 28-40 weeks gestation, are particularly active for the growth of the fetal eye. The blood supply to the retina starts at the optic nerve at 16 weeks of gestation, and the vessels gradually grow out over the surface of the retina. The vessels reach the anterior edge of the retina and stop their progression at about the time of birth. Therefore, prematurely born baby's retinal vessels may be incompletely formed and poorly drain leading to blindness.

Wallace et al. 9] 4] have shown that ROP can be accurately and quickly detected by looking at the vessels within the retina. Especially, they demonstrate that tortuosity and dilation of retinal vessels are good predictors for ROP. Their system uses a lens to magnify a baby's dilated pupil and a video capture system. After the procedure, physicians compare the video images with 5 reference images, Fig.1 which are graded 0 to 4 . Due to subjectivity of the comparison, this method can take as long as one hour per baby and, for some cases, there is a high inter-physician disagreement. 
We have developed a new tool for evaluating retinopathy of prematurity. Our system includes a computer-aided diagnosis software which assess dilation and tortuosity automatically. We have also used digital video recording and speech recognition to facilitate the integration into the clinic. Our system is described next.
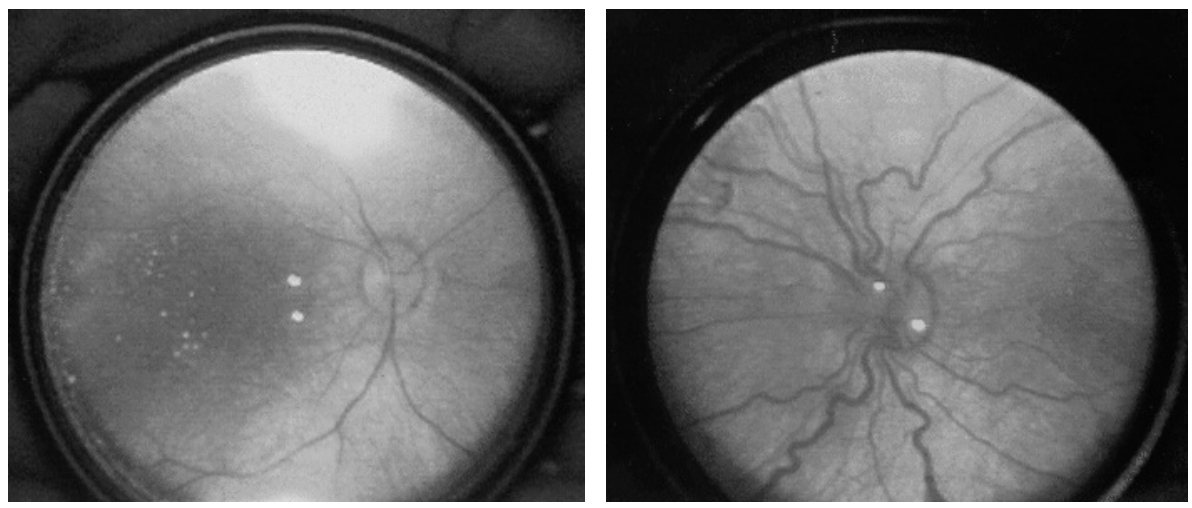

Fig. 1. Reference images for evaluation of retinopathy of prematurity. Grade 0(left) represents normal vessels and Grade 4(right) shows ROP

\section{Methods}

\subsection{Overview of the System}

Our system is composed of 4 parts: a lens, a digital video acquisition system, a speech recognition and image processing software. The lens is used to magnify the view through the baby's dilated pupil in order to improve the visibility of blood vessels. Radial distortions are minimal and at most minimally bias the image; the same lens is used to acquire the reference images. The digital acquisition system is composed of a head-mounted camera, Fig[2-left, which records the image the physician sees. The camera is composed of an incident light beam and two mirrors that coincide to form a monocular digital view. During the procedure the camera is connected to a laptop computer which can digitize still images from the video signal. The light beam from the camera and the lens can combine to introduce glare, and without precaution, the digitized image may be useless, Fig 2 right. Therefore, a real-time glare detector has been implemented; it produces a beep when too much glare is present in the live video.

Another important feature of our system is the addition of a speech recognition engine 7] [8]. In the clinic, the physician is often alone when capturing the images. Wearing a microphone clipped to their jacket, the physician needs only 

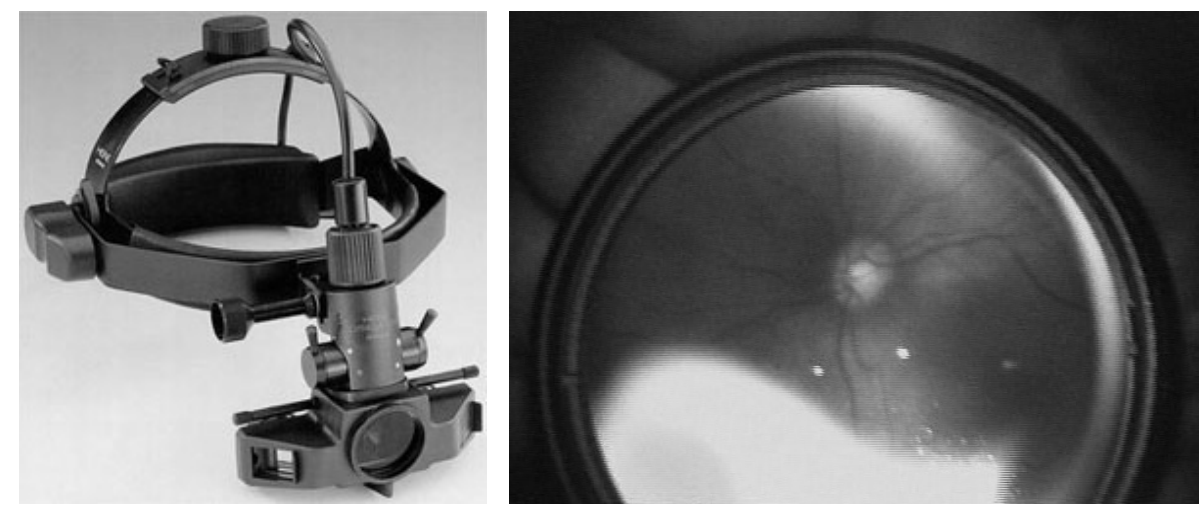

Fig. 2. The OMEGA 100 Head-worn indirect Ophthalmoscope from Heine Optotechnik(left). The light beam from the head-mounted camera reflects on the lens and produce glare(right)

to say "capture" to digitize an image. At the same time the glare detector gives feedback on the quality of the image. As the image is captured, the retinopathy grade estimation is computed in less than 30 seconds, much less than the time required to go from one baby to the next in the pediatric intensive care unit.

The estimation of the disease is done using a vessel segmentation method. This is described in the next section.

\subsection{Vessel Quantification}

Retinopathy of Prematurity diagnosis is based upon visual inspection of retinal blood vessels. Martinez-Perez et al. 6] have developed a region growing technique combined with scale-space analysis to extract blood vessels in the retina. Leandro et al. [5] use a continuous wavelet transform combined with morphological operators to segment blood vessels within the retina. This last method does not use any model information and the output of the algorithm is a binary image representing the segmentation. On the other hand, our method uses geometric information by considering blood vessels as tubes and better supports more complex measures on the extracted data such as tortuosity and dilation. Based on the extracted vessels, the four quadrants of the retina are identified and then grade is defined via classification using a trained neural network. The retina is usually composed of four major veins and four major arteries so that one major vein and one major artery are coupled together. We use this information to define the four quadrants.

The vessel extraction is based on the work of Aylward et al. [1] which extracts blood vessels from 3-dimensional images using a scale space technique with subvoxel accuracy. Our algorithm traverses a ridge in an intensity function $\boldsymbol{F}$, using the Hessian at a point $x$. We define $\alpha$ and $\beta$ as ascending-ordered eigenvalues of the Hessian at $x, \boldsymbol{u}$ and $\boldsymbol{v}$ as the corresponding eigenvectors of the Hessian, and 
$\boldsymbol{P}$ and $\boldsymbol{Q}$ as the directional derivatives: $\boldsymbol{P}=\boldsymbol{u} \cdot \boldsymbol{F}$ and $\boldsymbol{Q}=\boldsymbol{v} \cdot \boldsymbol{F}$. Therefore, if $x$ is exactly in the middle of the ridge the following conditions must hold: $\alpha$ $<0$ and $\boldsymbol{P}=0$. Given an initial starting point close to the ridge, the intensity ridge is computed to minimize $\boldsymbol{P}$ using a direction search with respect to the Hessian. The line search is performed from $x$ in the direction $\boldsymbol{u}$ to find the local minimum of $\boldsymbol{P}$. If the resulting minimum is not within a specified tolerance a new initial point is required.

The initial starting points are defined by looking at minimum intensity values along a circle centered on the optic nerve where vessels have low intensity values comparing to the background. The diameter of the circle is chosen to be approximately $30 \%$ of the size of the lens, . We assume that the optic nerve is located close to the center of the lens, since it must be there for the image to be valid. Thus, a 2-dimensional Hough transform is used to detect the lens and the approximate center. Note that the initial starting point can be anywhere along the ridge, i.e., it does not have to located at one of the ends of the vessel.

When the ridge is found, a ridge traversal technique is used to extract the vessel. The ridge is traversed by explicitly computing the tangent direction at $x$ and therefore uses third derivative information. In order to maintain $C^{0}$ continuity of the third derivative, quadric splines interpolation is used. The algorithm follows the tangent direction $\boldsymbol{v}$ and if the new point is too far from the ridge the flow technique is used to get on the ridge. We use a 0.1 voxel units as step size.

The width estimation is done by maximizing the medialness function $M(x, \sigma)$ at point $x$ at scale $\sigma$ over the image $I$. This medialness function incorporates a 2D gaussian $G(x ; \sigma)$.

$$
M(x ; \sigma)=-\sigma^{2} \nabla^{2} G(x ; \sigma) * I(x)
$$

Therefore, an estimate of the radius at each point $x$ along the centerline of the vessel is known and defines, after averaging, the dilation factor for the specified vessel. Tortuosity $\tau$ is defined as the ratio by which the straight distance between the two end points $\left(x_{0}, x_{1}\right)$ of the considered vessel is smaller than the geodesic distance $l$ of the segmented model.

$$
\tau=\frac{\left\|x_{1}-x_{0}\right\|}{l}
$$

After tortuosity and dilation estimations, quadrants are defined using the distance between vessels to form 4 clusters.

\section{Results}

Our algorithm has been tested on 20 premature infants, 10 normals and 10 representing various degrees of dilation and tortuosity. These 20 posterior pole images were analyzed by both our algorithm and two masked examiners experienced in the diagnosis of ROP. The standard photograph, Fig 3, obtained from the CRYO-ROP study[2] representing the minimum degree of dilation and 


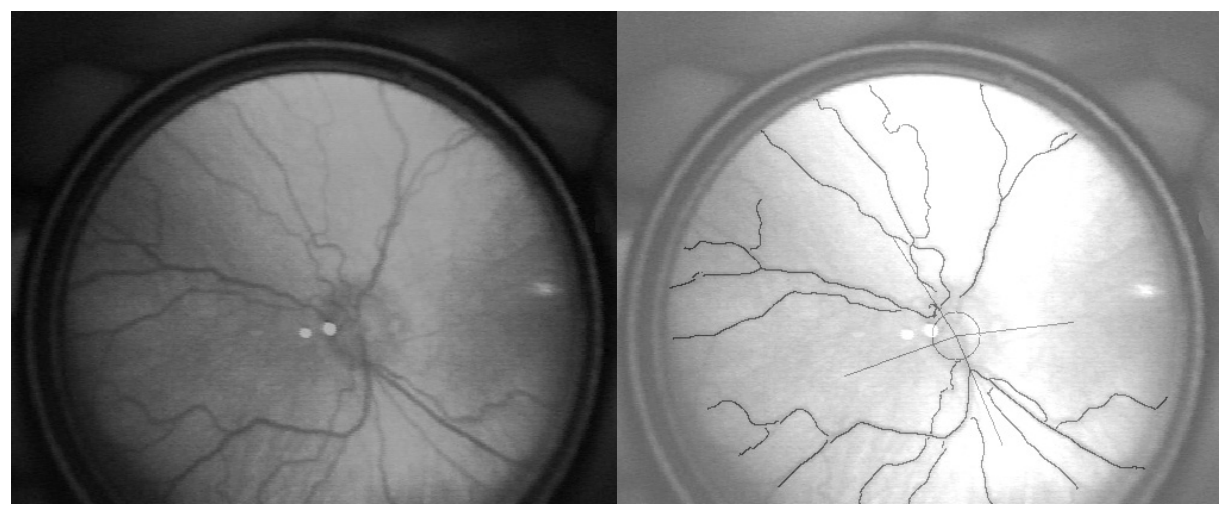

Fig. 3. Image captured from our acquisition system(left) and the resulting segmentation of blood vessels(right)

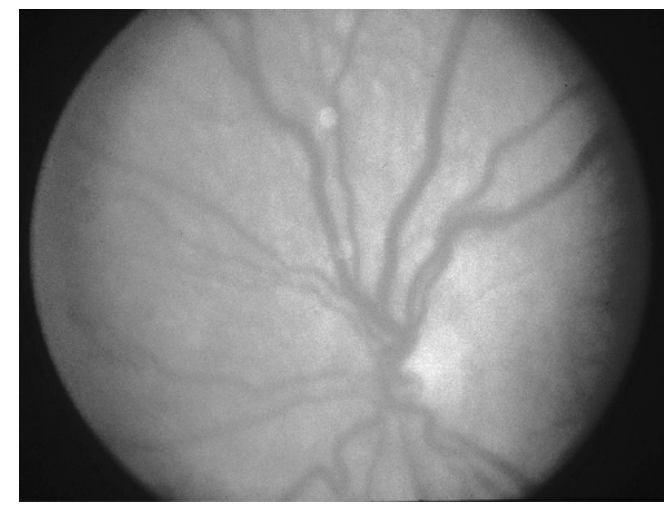

Fig. 4. Standard photograph obtained from the CRYO-ROP study

tortuosity required for plus disease was also digitized, analyzed, and used as a numeric threshold for the automated determination of plus disease.

The examiners agreed on the presence or absence of plus disease in 17 of 20 cases. Of the 5 images determined to have plus disease by both examiners, 4 were calculated to have plus disease by our algorithm ( $80 \%$ sensitivity). Of the 12 images without plus disease, 11 were computed to not have plus disease $(92 \%$ specificity). The examiners disagreed on the presence of plus disease on 3 images, and all 3 were calculated by our method to have dilation and tortuosity which was greater than normal but insufficient for plus disease. Fig [5 and Fig [6]report the results obtained. The quantification of plus disease is determined positive if at least 2 of the 4 quadrants have higher tortuosity and dilation than the reference image. It is important to consider both dilation and tortuosity in the estimation process. The reference image has been normalied to have an average 
tortuosity and dilation of 10. Each images are then normalized with respect to the reference image.

\begin{tabular}{cccc} 
& Normal & Pre-plus disease & Plus disease \\
\hline $\begin{array}{c}\text { Number of images } \\
\begin{array}{c}\text { Number determined by computer } \\
\text { to have plus disease }\end{array}\end{array}$ & 11 & 3 & 5 \\
\hline $\begin{array}{c}\text { Number with }>2 \text { quadrants with }>10 \\
\text { tortuosity units }\end{array}$ & 2 & 0 & 4 \\
\hline $\begin{array}{c}\text { Number with }>2 \text { quadrants with }>10 \\
\text { dilation units }\end{array}$ & 5 & 1 & 4 \\
\hline $\begin{array}{c}\text { Average tortuosity } \\
\text { (tortuosity units) }\end{array}$ & 6.5 & 11.3 & $15.5(\mathrm{p}=0.001)$ \\
$\quad \begin{array}{l}\text { Average dilation } \\
\text { (dilation units) }\end{array}$ & 10.3 & 10.4 & $11.2(\mathrm{p}=0.12)$ \\
\hline $\begin{array}{l}\text { Average number of quadrants with }>10 \\
\text { tortuosity units }\end{array}$ & 0.6 & 1.7 & 3.0 \\
\hline $\begin{array}{l}\text { Average number of quadrants with }>10 \\
\text { dilation units }\end{array}$ & 1.5 & 1.7 & 2.8
\end{tabular}

Fig. 5. Results obtained on 20 children prematurely born

Examiners

\begin{tabular}{ccccc}
\hline & & Plus disease & Not plus disease & Total \\
\hline Computer & Plus disease & 4 & 1 & 5 \\
\hline & Not plus disease & 1 & 10 & 11 \\
\hline & Total & 5 & 11 & 16
\end{tabular}

Fig. 6. Examiner vs. Computer quantification. Our algorithm shows $80 \%$ sensitivity and $92 \%$ specificity

\section{Discussion and Conclusions}

Our Retinopathy of Prematurity detection system shows the same accuracy as experts and the whole procedure runs in less than 3 minutes. The integration in the clinic, by adding speech recognition and glare detection systems, has been shown to be a useful help for physicians. The overall time was decreased by $70 \%$. Our software was implemented using the Insight Toolkit[3]. Aspects of this work have been licensed (patent pending) to Medtronic Inc. (Minn, MN) and R2 Technologies (Los Altos, CA). 


\section{References}

1. Aylward S, Bullitt E: Initialization, Noise, Singularities, and Scale in Height-Ridge Traversal for Tubular Object Centerline Extraction IEEE Transactions on Medical Imaging, Feb, 2002, Pages 61-75

2. Cryotherapy for Retinopathy of Prematurity Cooperative Group: Multicenter trial of cryotherapy for retinopathy of prematurity: Preliminary results Arch Ophthalmos 1988;106:471-9

3. The Insight Toolkit: National Library of Medicine Insight Segmentation and Registration Toolkit. http://www.itk.org

4. Kylstra JA, Freedman SF, Capowsky JJ, Hall JG: Measurement of retinal vessel tortuosity as a means of diagnosis plus disease in ROP Invest Ophthalmol Vis Sci 1995:36:77

5. Leandro JJG, Cesar RM Jr,Jeline HF: Blood Vessels Segmentation in Retina: Preliminary Assessment of the Mathematical Morphology and of the Wavelet Transform Techniques SIBGRAPI 2001, XIV Brazilian Symposium on Computer Graphics and Image Processing, 15-18 October 2001, Florianópolis, Brazil

6. Martinez-Perez ME, Hughes AD, Stanton AV, Thom SA, Bharath AA, Parker KH: Retinal Blood Vessel Segmentation by means of Scale-Space Analysis and Region Growing Proceedings of the Second International Conference on Medical Image Computing and Computer Assisted Intervention, Cambridge, 1999.

7. Microsoft Speech Technologies: http://www.microsoft.com/speech/

8. Jomier J: Speech recognition library http://caddlab.rad.unc.edu/software

9. Wallace DK, Kylstra JA, Chesnutt DA: Prognostic Significance of Vascular Dilation and Tortuosity Insufficient for Plus Disease in Retinopathy of Prematurity American Association for Pediatric Ophthalmology and Strabismus, 1999.

Further demos at caddlab.rad.unc.edu.

Supported in part by Whitaker Foundation grant RG010341, and NLM N01 LM03501 